\title{
The structure of microbial populations in Nelore GIT reveals inter-dependency of methanogens in feces and rumen
}

\author{
Bruno G. N. Andrade ${ }^{1}$, Flavia A. Bressani ${ }^{1}$, Rafael R. C. Cuadrat ${ }^{2}$, Polyana C. Tizioto ${ }^{3}$, Priscila S. N. de Oliveira'
} Gerson B. Mourão ${ }^{4}$, Luiz L. Coutinho ${ }^{4}$, James M. Reecy ${ }^{5}$, James E. Koltes ${ }^{5}$, Paul Walsh ${ }^{6}$, Alexandre Berndt ${ }^{1}$, Julio C. P. Palhares ${ }^{1}$ and Luciana C. A. Regitano ${ }^{1 *}$

\begin{abstract}
Background: The success of different species of ruminants in the colonization of a diverse range of environments is due to their ability to digest and absorb nutrients from cellulose, a complex polysaccharide found in leaves and grass. Ruminants rely on a complex and diverse microbial community, or microbiota, in a unique compartment known as the rumen to break down this polysaccharide. Changes in microbial populations of the rumen can affect the host's development, health, and productivity. However, accessing the rumen is stressful for the animal. Therefore, the development and use of alternative sampling methods are needed if this technique is to be routinely used in cattle breeding. To this end, we tested if the fecal microbiome could be used as a proxy for the rumen microbiome due to its accessibility. We investigated the taxonomic composition, diversity and inter-relations of two different GIT compartments, rumen and feces, of 26 Nelore (Bos indicus) bulls, using Next Generation Sequencing (NGS) metabarcoding of bacteria, archaea and ciliate protozoa.

Results: We identified 4265 Amplicon Sequence Variants (ASVs) from bacteria, 571 from archaea, and 107 from protozoa, of which 143 (96 bacteria and 47 archaea) were found common between both microbiomes. The most prominent bacterial phyla identified were Bacteroidetes (41.48\%) and Firmicutes (56.86\%) in the ruminal and fecal microbiomes, respectively, with Prevotella and Ruminococcaceae UCG-005 the most relatively abundant genera identified in each microbiome. The most abundant archaeal phylum identified was Euryarchaeota, of which Methanobrevibacter gottschalkii, a methanogen, was the prevalent archaeal species identified in both microbiomes. Protozoa were found exclusively identified in the rumen with Bozasellal Triplumaria being the most frequent genus identified. Co-occurrence among ruminal and fecal ASVs reinforces the relationship of microorganisms within a biological niche. Furthermore, the co-occurrence of shared archaeal ASVs between microbiomes indicates a dependency of the predominant fecal methanogen population on the rumen population.

(Continued on next page)
\end{abstract}

\footnotetext{
* Correspondence: luciana.regitano@embrapa.br

${ }^{1}$ Embrapa Southeast Livestock, São Carlos, Brazil

Full list of author information is available at the end of the article
}

(c) The Author(s). 2020 Open Access This article is distributed under the terms of the Creative Commons Attribution 4.0 International License (http://creativecommons.org/licenses/by/4.0/), which permits unrestricted use, distribution, and reproduction in any medium, provided you give appropriate credit to the original author(s) and the source, provide a link to the Creative Commons license, and indicate if changes were made. The Creative Commons Public Domain Dedication waiver (http://creativecommons.org/publicdomain/zero/1.0/) applies to the data made available in this article, unless otherwise stated. 


\begin{abstract}
(Continued from previous page)
Conclusions: Co-occurring microorganisms were identified within the rumen and fecal microbiomes, which revealed a strong association and inter-dependency between bacterial, archaeal and protozoan populations of the same microbiome. The archaeal ASVs identified as co-occurring between GIT compartments corresponded to the methanogenic genera Methanobrevibacter and Methanosphaera and represented $26.34 \%$ of the overall archaeal sequencesdiversity in the rumen and $42.73 \%$ in feces. Considering that these archaeal ASVs corresponded to a significant part of the overall diversity of both microbiomes, which is much higher if one includes the interactions of these co-occurring with other rumen archaea ASVs, we suggest that fecal methanogens could be used as a proxy of ruminal methanogens.
\end{abstract}

Keywords: Archaea, Bacteria, Bos indicus, Metabarcoding, Methanobrevibacter, Microbiome, Microbiota

\section{Background}

Nelore (Bos indicus) is a beef breed adapted to tropical environments and constitutes most of the biggest commercial herd in the world, the Brazilian bovine herd. Beef production is a fundamental part of the Brazilian economy. It represents $6 \%$ of the gross domestic product (GDP), with more than 1.6 million tons of beef exported worldwide in 2018 (http://www.abiec.com.br).

Ruminants, such as Nelore, require an abundant and diverse ruminal microbiota in order to digest complex polysaccharides, such as cellulose. This community produces enzymes capable of breaking down these polysaccharides into short-chain fatty acids (SCFAs), that provide the host with nutrients and energy required for its development and maintenance through enteric fermentation [1,2]. Fermentation also produces methane as a by-product, which is a greenhouse gas that contributes 28 times more to climate change than carbon dioxide, through the action of methanogenic archaea [3], also known as methanogens. These microorganisms are responsible for the production of $7-18 \%$ of greenhouse gases of anthropogenic origin and the loss of up to 2$12 \%$ of the total energy ingested, therefore, also negatively affecting animal productivity [4-6].

Several studies that aimed to characterize the gastrointestinal tract (GIT) microbiota and its genetic material, the microbiome, of ruminants using culture-independent approaches have been published over the years, including studies of Brazilian Nelore cattle [7-9]. One limitation to these studies is the difficulty in sampling rumen digesta in the routine of cattle breeding, which can be achieved only by oral intubation, rumenocentesis, fistulation, or after slaughter. These methods are not conducive to effectively monitoring the cattle microbiome without generating stress or compromising the production system. Alternatives, such as the use of oral samples as a proxy for the rumen microbiome, have been proposed [10] and shown to be good predictors for the bacteria community, but not for archaea. Other sampling alternatives for monitoring the GIT microbiome, such as colon/fecal are poorly investigated or limited to a small number of animals [11], despite being identified as crucial in other mammals [12-14].

To fill some of these gaps and to propose new sampling strategies, this study aimed to characterize microbial populations from two distant sections of the Nelore GIT, the rumen and rectal ampulla, and to search for co-occurring patterns within and between these microbiomes.

\section{Methods \\ Experimental design, sample collection and processing}

Fecal and ruminal samples were collected from a population of 26 Brazilian Nelore bulls, born in 2014 and slaughtered in 2016, with an average age of 1 year and 11 months. The experiment was carried out at Embrapa Southeast Livestock and lasted 105 days, of which 15 days were exclusively utilized for animal adaptation to the feedlot. The diet consisted of corn silage (82\%), corn grains $(11.83 \%)$, soy grains $(4.69 \%)$, mineral supplements (1.48\%), active dry yeast, virginiamycin and monensin. Approximately $10 \mathrm{~g}$ of feces was collected from the rectal ampulla of each animal two weeks before slaughtering, kept on ice for approximately $2 \mathrm{~h}$ and stored at $-80^{\circ} \mathrm{C}$. About $50 \mathrm{~mL}$ of rumen content was collected from each animal immediately after slaughter, frozen in liquid nitrogen and stored at $-80^{\circ} \mathrm{C}$.

DNA extractions from ruminal and fecal samples were performed by using the Quick-DNA ${ }^{\mathrm{ma}}$ Fecal/Soil Microbe Miniprep Kit (ZYMO Research Corp., Irvine, CA) using $150 \mathrm{mg}$ of each sample, as stated by the standard protocol. PCR amplification of the bacterial and archaeal $16 \mathrm{~S}$ rRNA genes and protozoa 18S rRNA gene were performed with the primers set described in Table 1. Additionally, barcode indexes were added to all samples to allow for sample multiplexing. Amplicons were pooled at equimolar ratios in sequencing libraries and sequenced by an Illumina MiSeq platform $(2 \times 250 \mathrm{bp})$ using the Illumina V3 sequencing kit at the ESALQ Genomics Center (Piracicaba, SP, Brazil). The sequence 
Table 1 Primer set used to amplify 165 and 18S hypervariable regions of Bacteria, Archaea and ciliate Protozoa

\begin{tabular}{llll}
\hline Identifier & Sequence & Target & Reference \\
\hline 341-b-S-17F & CCTACGGGNGGCWGCAG & Bacteria & {$[15]$} \\
785-a-A-21R & GACTACHVGGGTATCTAATCC & Bacteria & {$[15]$} \\
Ar915aF & AGGAATTGGCGGGGGAGCAC & Archaea & {$[16]$} \\
Ar1386R & GCGGTGTGTGCAAGGAGC & Archaea & {$[16]$} \\
Reg1320R & AATTGCAAAGATCTATCCC & Protozoa & {$[2]$} \\
RP841F & GACTAGGGATTGGARTGG & Protozoa & {$[2]$} \\
\hline
\end{tabular}

data is available from the Sequence read archive (SRA) [accession number PRJNA525838].

\section{Data pre-processing and analysis}

Raw reads were quality checked, filtered for quality (>Q25) and trimmed at the positions 220 (forward) and 175 (reverse) based on aggregated quality plots generated by QIIME 2 (version 2018.8) [17]. The remaining data were submitted to DADA2 to resolve the sequences into Amplicon Sequence Variants (ASVs), instead of traditional Operational taxonomic units (OTUs) [18], which presents an improved taxonomic resolution and consistency. Additionally, chimeric sequences were excluded using the DADA2 algorithm.

Bacterial sequences were classified using the SILVA database version 132 [19], archaeal sequences using the Rumen and Intestinal Methanogen database (RIM-DB) [20] and protozoa sequences using a curated database [21]. Rarefaction curves were generated for each dataset and used to standardize the data (Additional file 1: Figure S1A, Additional file 2: Figure S2A). The resulting ASV table was used to determine alpha (Number of ASVs and Shannon-Wiener index) and beta diversities (Unweighted Unifrac distance) with QIIME 2.

\section{Statistical analysis}

In order to identify differences in the microbial community structure, alpha and beta diversities were contrasted using nonparametric statistical methods Kruskal-Wallis and PERMANOVA, respectively.

To be able to identify co-occurrence patterns among microorganisms, the correlation between their abundances was inferred using the SparCC algorithm, a statistical approach developed for sparse and compositional data, with 1000 bootstrap replicates [22, 23]. This algorithm applies a Bayesian model to estimate the actual fractions from observed counts and infer the Pearson correlation of logratio variances of ASV fractions. To avoid spurious correlations, only ASVs identified in 10\% of the samples with at least 100 sequences in total and $|r|>0.6$ were considered for intra microbiome analysis. Also, due to the reduced number of ASVs common to both microbiomes, only those with 10 sequences in total and $|r|>0.5$ were considered for the inter microbiome analysis. Principal coordinate analysis $(\mathrm{PCoA})$ with unweighted unifrac distance was performed by QIIME 2 and used to identify variables that stratify the samples. Co-occurrence networks were generated using the software Cytoscape [24].

\section{Results \\ Microbiome composition}

Sequencing of bacterial, archaeal, and protozoa amplicons from the rumen and fecal samples of 26 animals yielded a total of $9,667,533$ paired-end reads $(5,361,879$ paired-end reads for bacteria, 2,706,672 for archaea and $1,598,982$ for protozoa). Quality control, denoising and chimera exclusion retained a total of $5,465,431$ sequences resolved in 13,680 ASVs. A total of 4943 ASVs (4265 for the bacteria, 571 for the archaea and 107 for protozoa datasets) were retained after the exclusion of singletons. Among the bacterial and archaeal ASVs identified, 143 were common to both microbiomes, which comprised 96/4265 (2.30\%) for the bacteria and 47/571 $(8.23 \%)$ for the archaea datasets. Rarefaction curves based on the alpha-diversity metrics of Shannon-Wiener (diversity) reached a plateau, which indicated that the sampling depth was adequate, and additional sequences would not likely result in additional features.

We were not able to identify protozoa $18 \mathrm{~S}$ rRNA amplicons in the fecal microbiome; therefore, the following analyses were performed exclusively on the bacteria and archaea datasets. To be able to compare the diversity of the microbiomes, the data was rarefied to 40,000 reads for the bacteria dataset and to 6000 reads for the archaea dataset. Comparisons between microbiomes using alphadiversity metrics (Number of ASVs and Shannon-Wiener indexes) revealed the bacterial diversity of the rumen microbiome to be significantly richer and diverse than the fecal microbiome $(P<0.01)$ (Additional file 1: Figure S1B, $C$, Additional file 2: Figure S2B, C). We found no significant difference between the number of archaeal ASVs in the rumen and fecal microbiomes. However, we found a significant difference between the Shannon-Wiener index of both environments, with the ruminal archaea population richer than the fecal population.

Moreover, comparisons of beta-diversity metric (Unweighted Unifrac distance), performed with Principal coordinates analysis (PCoA) and PERMANOVA, showed two spatially separated and significant clusters (adjusted $P<0.01$ ) that corresponded to the rumen and fecal microbiomes (Additional file 1: Figure S1D, Additional file 2: Figure S2D).

\section{Taxonomic composition of bacteria in ruminal and fecal microbiomes}

A total of 19 phylum, 31 classes, 55 orders, 93 families and 222 genera were identified in the rumen and fecal microbiomes. At the phylum level, Bacteroidetes 
$(41.94 \% \pm 3.39 \%)$ was the most relatively abundant population in the rumen microbiome, followed by Firmicutes $(36.81 \% \pm 2.90 \%)$, Proteobacteria $(6.28 \% \pm 3.51 \%)$ and Spirochaetes $(4.80 \% \pm 1.41 \%)$. Conversely, Firmicutes was the most abundant phylum in fecal microbiomes $(56.67 \% \pm 5.08 \%)$, followed by Bacteroidetes $(27.65 \% \pm$ $3.96 \%)$, Proteobacteria $(11.76 \% \pm 6.21 \%)$ and Tenericutes $(1.50 \% \pm 1.02 \%)$.

At the genus level, Prevotella $(16.81 \% \pm 3.54 \%)$, Christensenellaceae R-7 (5.59\% $\pm 1.55 \%)$, Rikenellaceae RC9 $(4.8 \pm 1.50 \%)$ and Treponema $(4.40 \% \pm 1.50 \%)$ were the most relatively abundant in the rumen microbiome. Conversely, the genera Ruminococcaceae UCG-005 $(10.85 \% \pm 3.68 \%)$, Succinivibrio $(7.86 \% \pm 2.71 \%)$, Bacteroides $\quad(7.76 \% \pm 1.79 \%), \quad$ Ruminococcaceae UCG-010 $(5.52 \% \pm 2.42 \%)$ and Rikenellaceae RC9 $(5.11 \% \pm 1.11 \%)$ were the most abundant in the fecal microbiome (Fig. 1).

\section{Taxonomic composition of archaea in ruminal and fecal microbiomes}

The archaeal diversity of both microbiomes was limited when compared to the bacterial community. It harbored a total of one phylum, three classes, three orders, three families, four genera and seven species. At the phylum level, Euryarchaeota was the only phylum identified in all samples using RIM-DB.

At the genus level, the rumen microbiome was populated by Methanobrevibacter $(69.46 \% \pm 3.73 \%)$, Methanosphaera $(2.91 \% \pm 0.76 \%)$, Methanomicrobium $(0.65 \% \pm$ $1.10 \%)$, and an uncultured genus from the Methanomassiliicoccaceae family $(22.85 \% \pm 3.65 \%)$. The fecal microbiome was populated by Methanobrevibacter $(88.36 \% \pm$ $2.56 \%)$ and Methanosphaera $(1.39 \% \pm 0.67 \%)$, being less diverse than the rumen microbiome. RIM-DB allowed us to assign ASVs identified as Methanobrevibacter into two dominant species clades, the Methanobrevibacter gottschalkii and Methanobrevibacter ruminantium, as well as other species of this genus, such as Methanobrevibacter smithii and Methanobrevibacter wolinii. These represent most of the archaeal diversity in the rumen (49.67\% for M. gottschalkii, $18.37 \%$ for M. ruminantium) and fecal microbiomes (76.96\% for M. gottschalkii, $9.70 \%$ for $M$. ruminantium).

Additionally, 47 out of 571 archaeal ASVs identified as common to both microbiomes represented part of the of the M. gottschalkii, M. ruminantium and Methanosphaera populations. These ASVs corresponded to 54.63\%, 36.62\%

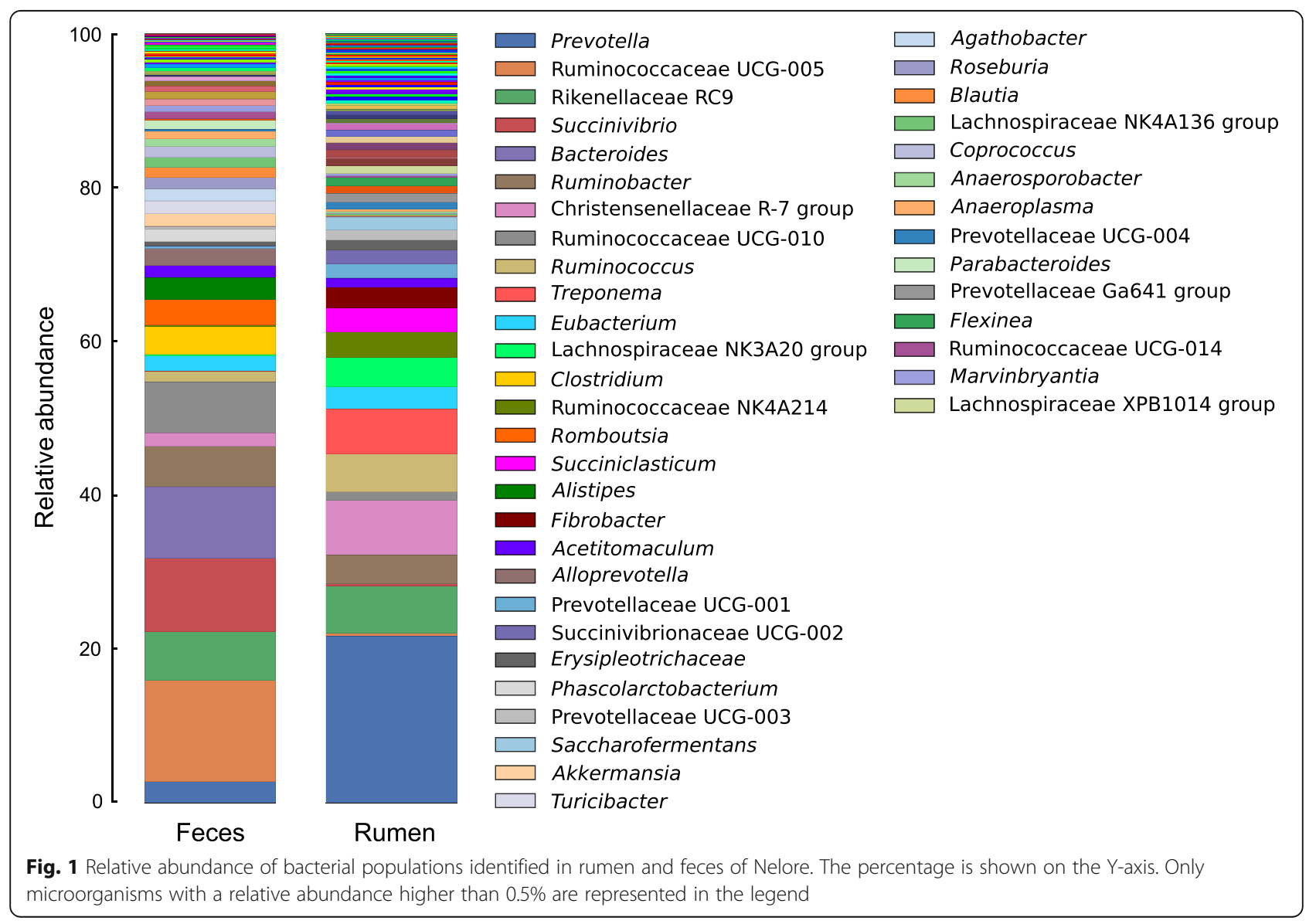


and $82.58 \%$ of these microorganisms' diversities in the rumen and $64.03 \%, 39.91 \%$ and $84.72 \%$ in the fecal microbiomes (Fig. 2a).

\section{Taxonomic composition of ciliate Protozoa}

The protozoa community of Nelore harbored a single phylum and class, two orders, three families and five genera. We identified the genus Bozasella/Triplumaria as the most abundant $(45.67 \% \pm 30.17 \%)$, with a relative abundance that ranged from $5.53 \%$ to $84.47 \%$ in the 26 rumen samples. The genus Entodinium was the second most abundant and prevalent genera, followed by uncharacterized protozoa from the Ophryoscolecidae and Isotrichidae families (Fig. 2b). The other families and genera accounted for less than $0.05 \%$ of the mean relative abundance.

\section{Co-occurrence patterns among microbial populations}

Correlations between the abundance patterns within and between bacteria, archaea and ciliate protozoa communities were tested to evaluate if these populations were interdependent, considering both the $|\mathrm{r}|$ threshold and significance defined in the methods section. A total of 1703 and 952 co-occurrences were identified in the fecal and ruminal microbiomes, respectively. These co-occurrences were used to generate co-occurrence networks (Additional file 2:
Figure S2, Additional file 3: Figure S3, Additional file 4: Figure S4).

Besides, 39 significant co-occurrences between ruminal and fecal archaea, of which nine identified as $M$. gottschalkii, one as M. ruminantium and three as Methanosphaera, co-occurred with the same ASV in the fecal microbiome (Fig. 3a). Conversely, eight significant cooccurrences between ruminal and fecal bacteria (Fig. 3b) were identified, but none between the same bacterial ASV.

\section{Discussion}

In recent years, culture-independent approaches have been applied to investigate microbial populations from the GIT of ruminants. Herein, we investigated the microbiome located in different sections of the Nelore cattle GIT, including bacteria, archaea and protozoa, and the relationship between these microorganisms, using metabarcoding. This study also characterized the distribution, relatedness, and co-occurrence of microbial populations within and between fecal and ruminal microbiomes.

\section{The structure of Nelore rumen and fecal microbiomes} Our results revealed that the ruminal and fecal microbiomes in Nelore cattle are significantly different based on both alpha and beta diversities. These results are in agreement with previous studies that reported the microbiome composition of GIT segments of bovines

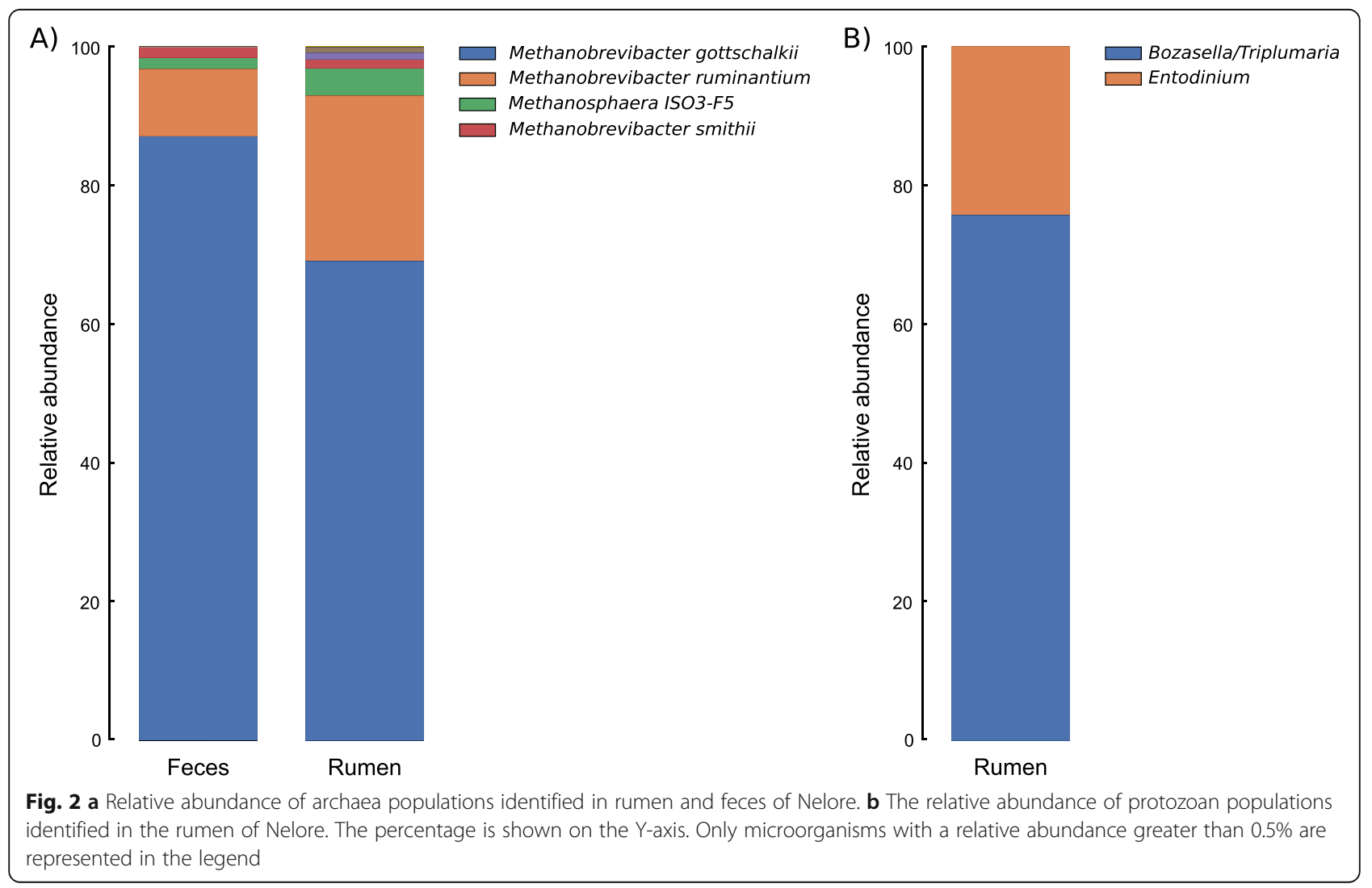




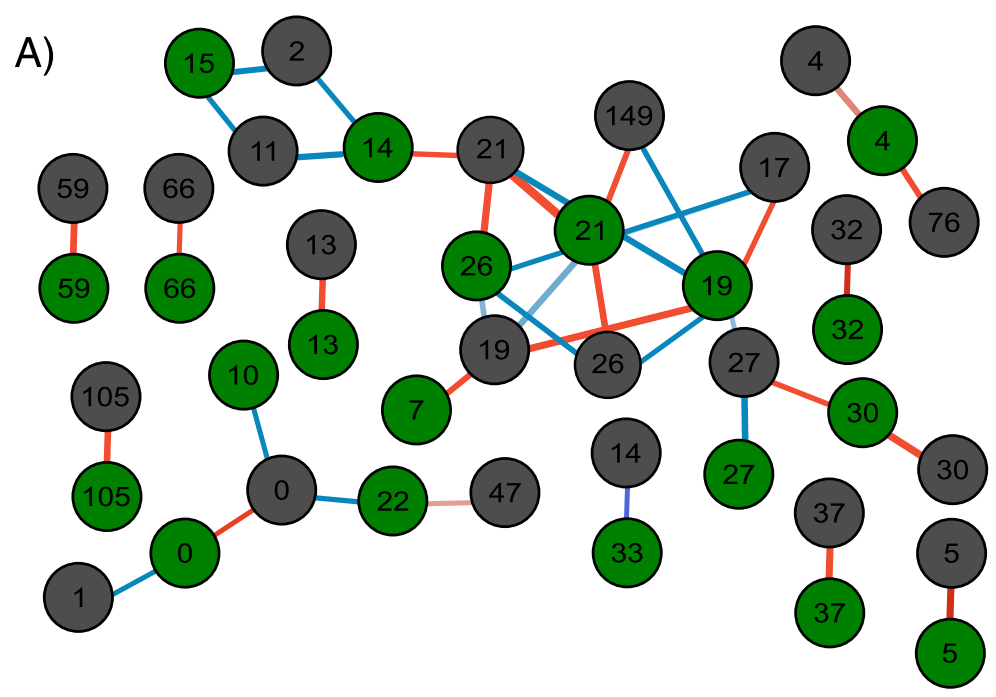

B)

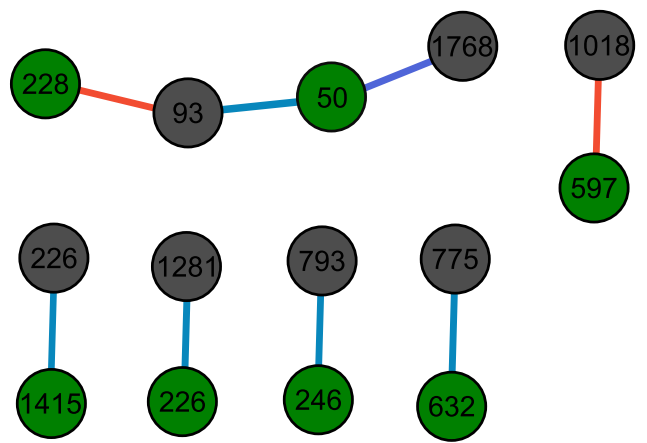

Fig. 3 Co-occurrence networks of ASVs common to ruminal (Green nodes) and fecal (Gray nodes) microbiomes, based on SparCC results. ASVs are represented by their respective numbers, with red edges representing positive correlation and blue, negative. a Eleven co-abundance networks with archaeal ASVs showing 12 positive correlations between the same ASV in different microbiomes. b Co-abundance networks with bacterial ASVs showing two positive correlations between the fecal and ruminal bacteria

$[10,14,25]$. Analysis of alpha-diversity metrics also revealed that the Nelore cattle rumen microbiome was richer and more diverse when compared to the fecal microbiome, which confirms previous results with Nelore cattle [9].

The Phylum Bacteroidetes dominated the Nelore rumen community (41.65\%), consistent with results from Bos taurus breeds such as Angus and Holstein, and other ruminant species [5, 25, 26]. Firmicutes (36.81\%) and Proteobacteria (6.27\%) also comprised a large proportion of the rumen microbiome. Differences in the ratio between Bacteroidetes and Firmicutes have been associated with obesity in humans [27] and milk-fat yield in Holstein Friesian cows [5]. At the genus level, Prevotella, a genus commonly found in the GIT of mammals and a significant player in carbohydrate metabolism and cellulose/hemicellulose degradation in ruminants, was identified as the most abundant genus, followed by Rikenellaceae RC9 and Christensenellaceae R-7, both previously reported in ruminants [28].
Firmicutes, the most prevalent phylum in the fecal microbiome, was represented primarily by the genera Ruminococcaceae UCG-005 and UCG-010, as previously described for post-weaning Holstein calves, Ayrshire cows and wild forest musk deer fecal microbiomes [10, 29, 30]. A single and abundant ASV, identified as Succinivibrio, was found in a substantially higher abundance in the fecal $(7.86 \%)$ than in the ruminal microbiome $(0.23 \%)$ of Nelore. This genus, of which the only described species is the Succinivibrio dextrinosolvens, is comprised of a rod-shaped bacterium that contributes to rumen starch digestion through the degradation of glucose. Furthermore, this genus is abundant in animals that are fed high starch diets [31].

The genus Methanobrevibacter was the most abundant archaea identified in both microbiomes, with the clades M. gottschalkii and M. ruminantium as the most prevalent. Strictly anaerobic microorganisms belonging to the archaea domain represent a small proportion of the 
overall taxonomic diversity in the rumen microbiome $[26,32,33]$. Most species of methanogenic archaea can use hydrogen, formate and methyl-compounds as their primary energy source, thereby reducing $\mathrm{CO}_{2}$ to $\mathrm{CH}_{4}$ in the rumen fermentation process.

Previous studies have shown that, rather than the absolute number of archaea, the contribution of individual methanogenic species is essential for $\mathrm{CH}_{4}$ production, especially the abundance of $M$. gottschalkii and M. ruminantium [34]. Recently, a study compared two groups of cows that were divergent for methane emission and found a higher relative abundance of $M$. gottschalkii in cows that were high $\mathrm{CH}_{4}$ emitters and of M. ruminantium in cows that were low emitters [34]. The authors suggested that this association could be due to differences in fermentation patterns of these methanogenic clades. Indeed, $M$. ruminantium M1 genome lacks the coding genes for methyl-CoM reductase II (McrII), having only genes for the isomeric form McrI [35]. McrI is expressed in low levels of $\mathrm{H}_{2}$ [36], which indicates that $M$. ruminantium is suited to thrive in an environment with low concentrations of $\mathrm{H}_{2}$, while $M$. gottschalkii is suited for high concentrations of $\mathrm{H}_{2}$.

Additionally, the genus Methanocorpusculum was not detected in any of the fecal samples used in this work, despite being identified (52\%) in the feces of five Ayrshire cows (52\%) [10] and Holstein cows (10.38 to 39.24\%) [36]. The addition of supplements, such as dry yeast, seems to play a negative role in the abundance of this genus in dairy cattle [37], which could contribute to its absence in our Nelore cattle microbiome. Thus, considering the scarce data on bovine fecal archaeal microbiome, our results reinforce the high dependency of the microbiome profile on breed, feed and environmental factors.

Regarding the rumen protozoa microbiome, the most abundant and prevalent genera identified in Nelore was the combination of the genera Bozasella and Triplumaria, because their respective 18S rRNA are 100\% identical. Both genera have been identified in Asian and African elephant intestines [38, 39]. Recently, these genera were reclassified to the unclassified family 1 (Uncf1) by Henderson et al and were not identified in the biggest survey of rumen microbiomes so far [2]. The reduced number of representative sequences in databases at the time the study was performed can explain this lack of results, or even the use of additives, such as monensin [40]. Also, the genus Entodinium, identified as the dominant protozoan genus in previous work and widespread in ruminants, was identified as the second most abundant genera and was present in all rumen samples analyzed in this study.

Although not essential for host survival, the rumen ciliate protozoa community is responsible for the release of vast amounts of hydrogen, contributing to methanogenic archaea metabolism and, therefore, methane production [41]. In fact, methane production drops by $11 \%$ when the host is defaunated, a process that removes the protozoa community, which can be taken as evidence of their importance in the methane production process [42].

\section{Co-occurrence of microbial populations suggests a close relationship among fibrolytic bacteria and methanogenic archaea}

Among the co-occurrent microorganisms, most are expected to be linked by metabolic processes, either synergistically in a mutualistic relationship, or through competition with organisms that share the same biological niche. For example, the ruminal ASV 33, classified as Succiniclasticum, a genus comprised by a single bacterium that ferments succinate into propionate, presented 11 connections in the cooccurrence network (3 positives, eight negatives). This ASV is co-occurring with ASVs identified as Prevotella, the most abundant genus in the rumen environment and a producer of succinate through the degradation of polysaccharides and glucose [43, 44]. In contrast, ASV 33 had a negative cooccurrence with three ASVs identified as Muribaculaceae (SparCC < - 0.6), a family of uncharacterized genera that are highly associated with the increase of propionate concentrations from succinate [45]. Thus, their negative correlation could be due to competition for substrates and nutrients.

The fecal ASV 1, classified as Ruminococcaceae UCG005, was identified as having the highest number of connections in the fecal co-occurrence network (33 positives and 29 negative correlations). This particular ASV is cooccurring with ASVs identified as Prevotella, Blautia and Parabacteroides, commonly found in the fecal microbiome and essential for polysaccharide degradation. However, ASV1 abundance was negatively correlated with other members of the Ruminococcaceae family, such as UCG-004, UCG-010 and UCG-005, which indicates a competition among species of this family. The others highly connected ASVs correspond to the genera Parabacteroides (ASV 36), Blautia (ASV 28) and an unknown genus from Lachnospiraceae family (ASV 62).

The archaea $M$. gottschalkii and $M$. ruminantium have different co-occurrence patterns with fibrolytic bacteria. M. gottschalkii co-occurred with bacteria from the genera Prevotella, Succiniclasticum and Ruminococus, while M. ruminantium co-occurred with bacteria from the family Lachnospiraceae and the genera Papilibacter and Acetitomaculum. Likewise, this difference was also observed in the fecal microbiome, 
in which the archaea M. gottschalkii co-occurred with bacteria from the genus Bacteroides, family Lachnospiraceae and Christensenellaceae, while $M$. ruminantium co-occurred with genera Ruminococcaceae UCG-005, Marvinbryantia and Blautia. Indeed, Kittelman et al. [13] demonstrated that the $M$. gottschalkii and $M$. ruminantium have different patterns of co-occurrence with fibrolytic bacteria in Bos taurus. However, this is the first time that this pattern is observed in the fecal microbiome.

The genera Bozasella/Triplumaria and Entodinium, the two dominant protozoan genera identified in Nelore, presented a strong and significant association with the archaea species $M$. gottschalkii and M. ruminantium and with fibrolytic bacteria, such as Prevotella and Fibrobacter [2]. Protozoa are known to be colonized by methanogens either as intracytoplasmic commensals or attached to their exterior surface $[42,46]$ and, although the symbiotic relationship between methanogens and protozoa is still unclear, our findings indicated a close relationship between these genera. Furthermore, the abundance of the genus Fibrobacter and some species of Prevotella are reduced in defaunated animals, suggesting that these genera may share a beneficial relationship with ciliate protozoa [47].

\section{Co-occurrence of ASVs inter-microbiomes indicates that fecal archaea as dependent on the rumen archaea population}

Among the 96 bacterial ASVs identified as common to both microbiomes, only eight significant pairwise cooccurrences were found, but none between the same ASV. Therefore, this indicates that despite having a considerable number of ASVs in common, the fecal and ruminal populations of bacteria are not co-occurring. Indeed, Tapio et al. [10] reported similar results with a different approach, in which only the bacterial taxa present in oral samples are similar to rumen samples. Studies have shown that bacteria colonize and persist in different environments, including the transfer of gut microbiome from mice to zebrafish $[48,49]$. This is evidence that the set of conditions in which these organisms can live, also known as the fundamental niche, is much larger than the conditions where the organism does live, or its realized niche [50]. The absence of cooccurrence of bacterial ASVs between microbiomes indicates that they successfully colonized both microbiomes, thus acquiring independent growth patterns according to each environmental condition.

On the other hand, of the 39/47 archaeal ASVs common to both environments co-occurred between microbiomes. Moreover, nine ruminal ASVs identified as $M$. gottschalkii, two as M. ruminantium and 4 as Methanosphaera had a strong and significant positive correlation with the same ASVs in the fecal microbiome. These cooccurrent ASVs represent $26.34 \%$ and $42.73 \%$ of the archaeal diversity in the rumen and feces microbiomes, respectively, which indicates that they are not typical residents of the gut/fecal environment but carried through the GIT. Also, these specific ruminal archaeal ASVs co-occurred with 19 highly abundant archaeal ASVs in the rumen, which corresponds to a total of $15.28 \%$ of the mean archaea abundance. Although new experiments should be performed to investigate further the relations between these microbiomes, such as shotgun metagenomic sequencing and quantitative PCR targeting these ASVs, these results indicate the potential use of the fecal archaea microbiome as a proxy for the ruminal archaea population in Nelore cattle.

\section{Conclusions}

The rumen and fecal microbiomes harbor structured populations with abundant microorganisms, whose cooccurrences may reflect their relationships. Archaeal ASVs identified as co-occurring between microbiomes corresponded to a significant part of the overall archaeal diversity, which in fact, is much higher if one includes the interactions of these co-occurring ASVs with other archaea within their own microbiomes. Therefore, we suggest that fecal methanogens could be used as a proxy for rumen methanogens.

\section{Supplementary information}

Supplementary information accompanies this paper at https://doi.org/10. 1186/s40104-019-0422-x

Additional file 1: Figure S1. A) Rarefaction curves of Bacterial 16S samples using the Shannon-Wiener index. B) Observed ASVs are significantly different among the environments when compared using the Kruskal-Wallis test. C) Shannon-Wiener index is significantly different among the environments when compared using the Kruskal-Wallis test. D) PCoA plot showing the stratification of the microbial populations in both environments.

Additional file 2: Figure S2. A) Rarefaction curves of Archaeal 165 samples using the Shannon-Wiener index. B) Observed ASVs are not significantly different among the environments when compared using the Kruskal-Wallis test. C) Shannon-Wiener index is not significantly different among the environments when compared using the Kruskal-Wallis test. D) PCoA plot showing the stratification of the microbial populations in both environments.

Additional file 3: Figure S3. A) Co-occurrence networks of bacterial ASVs identified in the ruminal microbiome. B) Co-occurrence networks of bacterial ASVs of the fecal. C) Co-occurrence networks of archaea ASVs identified in the ruminal microbiome. D) Co-occurrence networks of archaea ASVs identified in the fecal microbiome. ASVs are represented by their respective numbers and their taxonomic information, from family to order, by colors. Red edges represent positive correlation and blue, negative. Edges widths are related to the strength of the correlation.

Additional file 4: Figure S4. Co-occurrence networks of interdomain ASVs (Bacteria, Archaea and Protozoa). A) rumen and B) fecal origin, based on SparCC results. ASVs are represented by their respective numbers and their taxonomic information, from family to order, by colors. Red edges represent positive correlation and blue, negative. Edges widths are related to the strength of the correlation. 


\section{Abbreviations}

ASV: Amplicon Sequence Variant; GIT: Gastrointestinal Tract; OTU: Operational Taxonomic Unit

\section{Acknowledgments}

We thank the São Paulo Research Foundation (FAPESP - 2017/12642-8) and the National Council for Scientific and Technological Development (CNPq 03.17.00.046.00.03.002) for providing financial support. We also thank Dr. Marcela Morelli for collecting sample data, Dr. Leandro Sannomiya Sakamoto and the staff of the Embrapa Southeast Cattle Animal biotechnology laboratory for assistance on sample collection.

\section{Authors' contributions}

BGNA, LCAR, AB, JCPP, JMR, LLC and JEK conceived the experiment; BGNA and $F A B$ performed the experiments; BGNA, RRCC and GBM performed analysis; BGNA, LCAR, RRCC, PSNO, PW and PCT interpreted the results; BGNA and LCAR drafted and revised the manuscript. All authors read and approved the final manuscript.

\section{Funding}

This study was conducted with funding from EMBRAPA, São Paulo Research Foundation scholarship to BGNA (grant number: 2017/12642-8), the National Council for Scientific and Technological Development (CNPq, grant number: 428153/2018) and fellowships to LCAR and LLC.

\section{Availability of data and materials}

All sequencing data are available in the NCBI Sequence Read Archive (SRA), under the bioproject number PRJNA525838.

\section{Ethics approval and consent to participate}

Experimental procedures were conducted following Brazilian guidelines on animal welfare and approved by the Ethics Committee on the Use of Animals, College of Veterinary and Animal Science, São Paulo State University under protocol $n^{\circ} 8510190118$

\section{Consent for publication}

Not applicable.

\section{Competing interests}

The authors Paul Walsh and Polyana C. Tizioto were employed by the companies Nsilico and NGS Genomic Solutions, respectively. All other authors declare that the research was conducted in the absence of any commercial or financial relationships that could be construed as a potential conflict of interest.

\section{Author details}

${ }^{1}$ Embrapa Southeast Livestock, São Carlos, Brazil. ${ }^{2}$ Department of Molecular Epidemiology, German Institute of Human Nutrition Potsdam-Rehbrücke (DIfE), Nuthetal, Germany. ${ }^{3}$ NGS Genomic Solutions, Piracicaba, Brazil. ${ }^{4}$ Department of Animal Science, University of São Paulo/ESALQ, Piracicaba, Brazil. ${ }^{5}$ Department of Animal Science, lowa State University, Ames, IA, USA. ${ }^{6}$ NSilico Life Science, Dublin, Ireland.

\section{Received: 13 September 2019 Accepted: 23 December 2019} Published online: 24 February 2020

\section{References}

1. Leahy SC, Kelly WJ, Ronimus RS, Wedlock N, Altermann E, Attwood GT. Genome sequencing of rumen bacteria and archaea and its application to methane mitigation strategies. Animal. 2013;7(Suppl 2):235-43.

2. Henderson G, Cox F, Ganesh S, Jonker A, Young W, Abecia L, et al. Rumen microbial community composition varies with diet and host, but a core microbiome is found across a wide geographical range. Sci Rep. 2015;5: 14567.

3. Johnson DE, Ward GM. Estimates of animal methane emissions. Environ Monit Assess. 1996;42:133-41.

4. Carberry CA, Kenny DA, Han S, McCabe MS, Waters SM. Effect of phenotypic residual feed intake and dietary forage content on the rumen microbial community of beef cattle. Appl Environ Microbiol. 2012;78:4949-58.
5. Jami E, White BA, Mizrahi I. Potential role of the bovine rumen microbiome in modulating milk composition and feed efficiency. PLoS One. 2014;9: e85423.

6. Kittelmann S, Pinares-Patiño CS, Seedorf H, Kirk MR, Ganesh S, McEwan JC et al. Two different bacterial community types are linked with the lowmethane emission trait in sheep. PLoS One. 2014;9:e103171.

7. Comtet-marre S, Parisot N, Leperca P, Chaucheyras-durand F, Mosoni P, Peyretaillade $\mathrm{E}$, et al. Metatranscriptomics reveals the active bacterial and eukaryotic fibrolytic communities in the rumen of dairy cow fed a mixed diet. Front Microbiol. 2017;8:67.

8. Granja-Salcedo YT, Fernandes RM, de Araujo RC, Kishi LT, Berchielli TT, de Resende FD, et al. Long-term encapsulated nitrate supplementation modulates rumen microbial diversity and rumen fermentation to reduce methane emission in grazing steers. Front Microbiol. 2019;10:614.

9. de Oliveira MNV, Jewell KA, Freitas FS, Benjamin LA, Tótola MR, Borges AC, et al. Characterizing the microbiota across the gastrointestinal tract of a Brazilian Nelore steer. Vet Microbiol. 2013;164:307-14.

10. Tapio I, Shingfield KJ, McKain N, Bonin A, Fischer D, Bayat AR, et al. Oral samples as non-invasive proxies for assessing the composition of the rumen microbial community. PLoS One. 2016;11:e0151220.

11. Noel SJ, Olijhoek DW, Mclean F, Løvendahl P, Lund P, Højberg O. Rumen and fecal microbial community structure of Holstein and Jersey dairy cows as affected by breed, diet, and residual feed intake. Animals. 2019;9:498.

12. Flint HJ, Duncan SH, Scott KP, Louis P. Interactions and competition within the microbial community of the human colon: links between diet and health. Environ Microbiol. 2007;9:1101-11.

13. Flint HJ, Bayer EA, Rincon MT, Lamed R, White BA. Polysaccharide utilization by gut bacteria: potential for new insights from genomic analysis. Nat Rev Microbiol. 2008;6:121-31.

14. Myer PR, Wells JE, Smith TPL, Kuehn LA, Freetly HC. Microbial community profiles of the colon from steers differing in feed efficiency. Springerplus. 2015:4:454 Springer International Publishing

15. Klindworth A, Pruesse E, Schweer T, Peplies J, Quast C, Horn M, et al. Evaluation of general $16 \mathrm{~S}$ ribosomal RNA gene PCR primers for classical and next-generation sequencing-based diversity studies. Nucleic Acids Res. 2013; 41:e1.

16. Kittelmann S, Seedorf H, Walters WA, Clemente JC, Knight R, Gordon II, et al Simultaneous amplicon sequencing to explore co-occurrence patterns of bacterial, archaeal and eukaryotic microorganisms in rumen microbial communities. PLoS One. 2013:8:e47879.

17. Bolyen E, Rideout JR, Dillon MR, Bokulich NA, Abnet CC, Al-Ghalith GA, et al. Reproducible, interactive, scalable and extensible microbiome data science using QIIME 2. Nat Biotechnol. 2019;37:852-7.

18. Callahan BJ, McMurdie PJ, Rosen MJ, Han AW, Johnson AJA, Holmes SP. DADA2: high-resolution sample inference from Illumina amplicon data. Nat Methods. 2016;13:581-3.

19. Quast C, Pruesse E, Yilmaz P, Gerken J, Schweer T, Yarza P, et al. The SILVA ribosomal RNA gene database project: improved data processing and webbased tools. Nucleic Acids Res. 2013:41:590-6.

20. Seedorf H, Kittelmann S, Henderson G, Janssen PH. RIM-DB: a taxonomic framework for community structure analysis of methanogenic archaea from the rumen and other intestinal environments. Peer J. 2014;2:e494.

21. Kittelmann S, Devente SR, Kirk MR, Seedorf $H$, Dehority BA, Janssen PH. Phylogeny of intestinal ciliates, including Charonina ventriculi, and comparison of microscopy and 185 rRNA gene pyrosequencing for rumen ciliate community structure analysis. Appl Environ Microbiol. 2015:81:2433-44.

22. Friedman J, Alm EJ. Inferring correlation networks from genomic survey data. PLoS Comput Biol. 2012;8:1-11.

23. Watts SC, Ritchie SC, Inouye M, Holt KE. FastSpar: rapid and scalable correlation estimation for compositional data. Bioinformatics. 2019;35:1064-6.

24. Su G, Morris JH, Demchak B, Bader GD. Biological network exploration with Cytoscape 3. Curr Protoc Bioinformatics. 2014;47:1-8.

25. Myer PR, Smith TPL, Wells JE, Kuehn LA, Freetly HC. Rumen microbiome from steers differing in feed efficiency. PLOS One. 2015;10:1-17.

26. Zhou Z, Fang L, Meng Q, Li S, Chai S, Liu S, et al. Assessment of ruminal bacterial and archaeal community structure in yak (Bos grunniens). Front Microbiol. 2017:8:1-10.

27. Clarke SF, Murphy EF, Nilaweera K, Ross PR, Shanahan F, O'Toole PW, et al. The gut microbiota and its relationship to diet and obesity: new insights. Gut Microbes. 2012;3:186-202. 
28. Xin J, Chai Z, Zhang C, Zhang Q, Zhu Y, Cao H, et al. Comparing the microbial community in four stomach of dairy cattle, yellow cattle and three yak herds in Qinghai-Tibetan Plateau. Front Microbiol. 2019;10:1547.

29. Li Y, Hu X, Yang S, Zhou J, Zhang T, Qi L, et al. Comparative analysis of the gut microbiota composition between captive and wild forest musk deer. Front Microbiol. 2017:8:1-10.

30. Dill-Mcfarland KA, Breaker JD, Suen G. Microbial succession in the gastrointestinal tract of dairy cows from 2 weeks to first lactation. Sci Rep. 2017;7:1-12.

31. O'Herrin SM, Kenealy WR. Glucose and carbon dioxide metabolism by Succinivibrio dextrinosolvens. Appl Environ Microbiol. 1993;59:748-55.

32. Liu C, Zhu ZP, Liu YF, Guo TJ, Dong HM. Diversity and abundance of the rumen and fecal methanogens in Altay sheep native to Xinjiang and the influence of diversity on methane emissions. Arch Microbiol. 2012;194:353-61.

33. Tapio I, Snelling TJ, Strozzi F, Wallace RJ. The ruminal microbiome associated with methane emissions from ruminant livestock. J Anim Sci Biotechnol. 2017:8:1-11.

34. Shi W, Moon CD, Leahy SC, Kang D, Froula J, Kittelmann S, et al. Methane yield phenotypes linked to differential gene expression in the sheep rumen microbiome. Genome Res. 2014;24:1517-25.

35. Leahy SC, Kelly WJ, Altermann E, Ronimus RS, Yeoman CJ, Pacheco DM, et al. The genome sequence of the rumen methanogen Methanobrevibacter ruminantium reveals new possibilities for controlling ruminant methane emissions. PLoS One. 2010;5:e8926.

36. Reeve JN, Nölling J, Morgan RM, Smith DR. Methanogenesis: genes, genomes, and who's on first? J Bacteriol. 1997:179:5975-86.

37. Jin $D$, Kang $K$, Wang $H$, Wang $Z$, Xue $B$, Wang $L$, et al. Effects of dietary supplementation of active dried yeast on fecal methanogenic archaea diversity in dairy cows. Anaerobe. 2017;44:78-86 Elsevier Ltd.

38. Ito A, Ishihara M, Imai S. Bozasella gracilis $\mathrm{n}$. sp. (Ciliophora, Entodiniomorphida) from Asian elephant and phylogenetic analysis of entodiniomorphids and vestibuliferids. Eur J Protistol. 2014;50:134-52.

39. Timoshenko O, Imai S. Eleven new ciliate species of the genus Triplumaria ( Ciliophora, Entodiniomorphida ) from Asian elephant, Elephas maximus and African elephant, Loxodonta africana. J Protozool Res. 1995;5:157-75.

40. Aowicki D, Huczyński A. Structure and antimicrobial properties of monensin $\mathrm{a}$ and its derivatives: summary of the achievements. Biomed Res Int. 2013; 2013:742149.

41. Newbold CJ, Lassalas B, Jouany JP. The importance of methanogens associated with ciliate protozoa in ruminal methane production in vitro. Lett Appl Microbiol. 1995;21:230-4.

42. Newbold CJ, De la Fuente G, Belanche A, Ramos-Morales E, McEwan NR. The role of ciliate protozoa in the rumen. Front Microbiol. 2015;6:1-14.

43. Pan $X$, Xue F, Nan $X$, Tang $Z$, Wang $K$, Beckers $Y$, et al. Illumina sequencing approach to characterize thiamine metabolism related bacteria and the impacts of thiamine supplementation on Ruminal microbiota in dairy cows fed high-grain diets. Front Microbiol. 2017;8:1818.

44. Takahashi N, Yamada T. Glucose metabolism by Prevotella intermedia and Prevotella nigrescens. Oral Microbiol Immunol. 2000;15:188-95.

45. Smith BJ, Miller RA, Ericsson AC, Harrison DC, Strong R, Schmidt TM. Changes in the gut microbiome and fermentation products concurrent with enhanced longevity in acarbose-treated mice. BMC Microbiol. 2019;19:130.

46. Guyader J, Eugène M, Nozière P, Morgavi DP, Doreau M, Martin C. Influence of rumen protozoa on methane emission in ruminants: a meta-analysis approach. Animal. 2014;8:1816-25.

47. Ozutsumi Y, Tajima K, Takenaka A, Itabashi H. Real-time PCR detection of the effects of protozoa on rumen bacteria in cattle. Curr Microbiol. 2006;52: $158-62$.

48. Seedorf H, Griffin NW, Ridaura VK, Reyes A, Cheng J, Rey FE, et al. Bacteria from diverse habitats colonize and compete in the mouse gut. Cell. 2014; 159:253-66.

49. Rawls JF, Mahowald MA, Ley RE, Gordon JI. Reciprocal gut microbiota transplants from zebrafish and mice to germ-free recipients reveal host habitat selection. Cell. 2006:127:423-33.

50. Hutchinson GE. Concluding remarks. Cold Spring Harb Symp Quant Biol. 1957:22:415-27.

\section{Ready to submit your research? Choose BMC and benefit from:}

- fast, convenient online submission

- thorough peer review by experienced researchers in your field

- rapid publication on acceptance

- support for research data, including large and complex data types

- gold Open Access which fosters wider collaboration and increased citations

- maximum visibility for your research: over $100 \mathrm{M}$ website views per year

At BMC, research is always in progress.

Learn more biomedcentral.com/submissions 